\title{
A New Linear Tire Model With Varying Parameters
}

\author{
Moad Kissai, Bruno Monsuez, Adriana Tapus \\ ENSTA ParisTech \\ Department of Computer and System Engineering \\ 828 Boulevard des Marchaux, \\ 91762 Palaiseau Cedex, France \\ e-mail: \{moad.kissai, bruno.monsuez, \\ adriana.tapus\}@ensta-paristech.fr
}

\author{
Didier Martinez \\ Groupe Renault \\ Chassis Systems Department \\ 1 Avenue du Golf, \\ 78280 Guyancourt, France \\ e-mail: didier.d.martinez@renault.com
}

\begin{abstract}
A large interest has recently been given to global chassis control. This requires a tire model adapted to control synthesis that can represent combined slip. In this paper, a new suitable tire model for vehicle coupled operations control is proposed. The existing models are either too simple to represent coupled behaviors or too complex to allow control synthesis. This new tire model takes benefits from both approaches, as it is linear with varying parameters. Simulations of coupled operations proved the model accuracy in the tire stable range and its promising potential for global chassis control synthesis.
\end{abstract}

Keywords- Tire Modeling, combined slip, linearization, global chassis control, AMESim (c) simulation.

\section{INTRODUCTION}

The development of chassis systems has been ongoing since the late 1970's [1]. Most of these systems are designed for single-objective performances. This is about to change with the arrival of autonomous vehicles. In fact, the virtual pilot has to handle combined operations. For example, let us consider the high speed cornering maneuver. In the present situation, the human pilot controls the longitudinal speed while active steering systems (e.g., the 4-Wheel Steering (4WS) system [2]) or, in hazardous situations, brake-based yaw control systems (e.g., The Electronic Stability Control (ESC) system [3]) control the lateral dynamics to ensure the vehicle stability. In contrast, the virtual pilot has to handle multi-objective control problems. Therefore, couplings should be taken into account.

One of the most important couplings is the combined slip as the tire is the only effector between the vehicle and the road [4]. In this context, two major approaches are adopted: empirical methods and theoretical methods. Figure 1 depicts the various differences (taken from [5]).

Six parameters are considered in [5]:

- Degree of fit: model's accuracy with respect to the design objective,

- Number of full scale tests: amount of tests required to validate the model,

- Complexity of formulations,

- Effort required to design the model,

- Insight in tire behavior: model's ability of predicting the tire behavior,

- Number of special experiments: amount of experiments required to develop the model.

As expected, empirical approaches require an important number of full scale tests with respect to theoretical approaches. These latter are more based on the tire's physical structure theory. Four categories are distinguished in [5]: "from experimental data only", "using similarity method", "through simple physical model", "through complex physical model". The first category at the extreme left uses regression procedures to develop mathematical formulations whose parameters fit best the measured data. A well-known empirical model is the Magic Formula [5]. This model provides an excellent fit for tire's efforts curves, which makes it more suitable for vehicle motion simulations. The similarity approach uses rather simple distortion and re-scaling methods to develop simpler empirical models. This method is particularly useful when fast computations are needed [5]. A good example would be Dugoff's model, which uses a simpler representation of tire deformation while keeping a good representation of combined slip [6]. But these two categories provide less insight in tire behavior. The relatively simple physical models of the third

\begin{tabular}{|c|c|c|c|}
\hline $\begin{array}{l}\text { from experimental } \\
\text { data only }\end{array}$ & $\begin{array}{l}\text { using similarity } \\
\text { method }\end{array}$ & $\begin{array}{l}\text { through simple } \\
\text { physical model }\end{array}$ & $\begin{array}{l}\text { through complex } \\
\text { physical model }\end{array}$ \\
\hline $\begin{array}{l}\text { fitting full scale } \\
\text { tyre test data } \\
\text { by regression } \\
\text { techniques }\end{array}$ & $\begin{array}{l}\text { distorting, } \\
\text { rescaling and } \\
\text { combining } \\
\text { basic } \\
\text { characteristics }\end{array}$ & $\begin{array}{l}\text { using simple } \\
\text { mechanical } \\
\text { representation, } \\
\text { possibly closed } \\
\text { form solution }\end{array}$ & $\begin{array}{l}\text { describing tyre } \\
\text { in greater detail, } \\
\text { computer simulation, } \\
\text { finite element } \\
\text { method }\end{array}$ \\
\hline
\end{tabular}
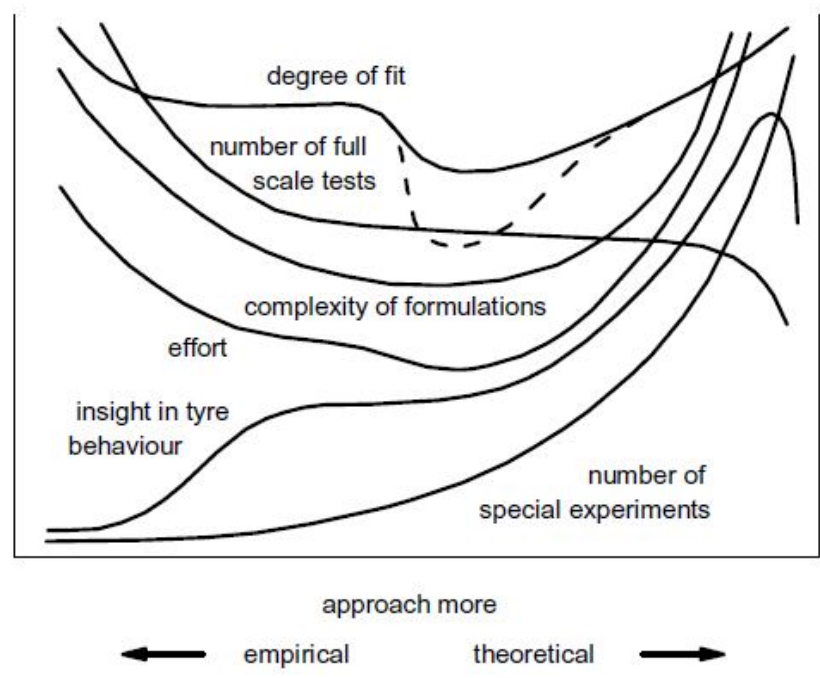

Fig. 1. Possible Categories of Tire Modeling Approaches [5]. 
category are more useful to get better understandings about the tire behavior. In this context, the "brush model" represents a good illustration [5], [7], [8]. Regarding the fourth category, interest is given to tire performances related to its construction. More detailed analysis is required and complex finite element based models are usually adopted [5].

On one hand, empirical models rely on experimental measures to make simulation more accurate, and on the other hand, theoretical models rely on physical models to give more insight about the tire behavior and improve its construction. No model however is designed for control synthesis. Physical models are too complex to be implemented in real-time operations, and empirical models use numerous parameters with poor physical meaning, which make them hard to measure in real time.

This paper outlines the design of a new tire model more adapted to control synthesis, and especially, global chassis control. For this purpose, the model should accurately represent the combined slip behavior in a simpler way using real-time measurable parameters. We propose consequently a new model based on Dugoff's model. This model is simple enough to be suitable for control synthesis as it is linear, and depends on measurable or estimable physical varying-parameters, which favor real-time implementation. The method adopted to develop the model is described in Section II. Results and interpretations are given in Section III. In Section IV, the model's relevance is discussed with respect to existing models. Conclusions and future works are consequently drawn at the end of this paper.

\section{DEVELOPMENT OF THE NEW TIRE MODEL}

First, we propose a set of requirements for the new tire model development. As far as global chassis control is concerned, the new model should:

- Respect the tire physical fundamentals to give enough insight into tire behavior,

- Represent as precise as possible combined slip behavior,

- Be simple enough for controllability issues,

- Depends on a minimum set of parameters that can be measured and estimated so as to favor real-time operations.

\section{A. Tire physical fundamentals}

The tire consists of a set of rubber blocks. Two friction mechanisms could be distinguished: shearing and sliding [9]. In shearing, the rubber block deforms without sliding with respect to the ground. A resistance force proportional to deformation is generated. When this force reaches a maximum value, called the Coulomb force, the rubber block rubs on the ground and start sliding. If slip value continue growing, the tire suffers from a loss of potential, which causes the instability of the vehicle.

The friction force maximum value is then very important to predict. It depends on two varying-parameters: the vertical load applied by the vehicle and the coefficient of friction that characterizes the condition of both the road and tire rubber [9].
Furthermore, longitudinal adhesion and lateral adhesion are competing: they must share the tire friction potential [9]. In fact, the overall adhesion is delimited by a "friction ellipse" [5], [7], [10]. A longitudinal force request would consequently penalize a lateral force request if the limits of adhesion are reached. Figure 2 summarizes the previous characteristics.

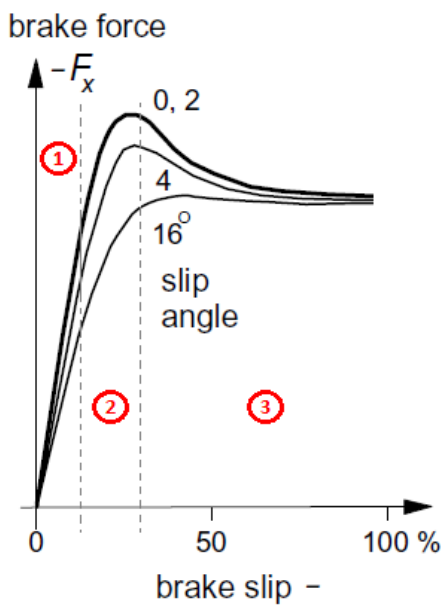

Fig. 2. Combined side force and brake force characteristics (adapted from [5]).

Three regions are distinguished:

- Region 1 : the curve is linear and increasing, the rubber blocks are deforming without sliding (shearing).

- Region 2 : the curve is nonlinear and increasing, and ends up reaching a maximum. A portion of the contact area begins to slide.

- Region 3 : the curve is decreasing, there is the sliding of the tire. The tire behavior is unstable.

The overall behavior of the tire is highly nonlinear.

\section{B. Existing tire models}

Three models have received large interest: The Magic Formula of Pacejka [5], the brush model [7],[8], and the physical model of Dugoff [6]. Next, SAE ISO notation [8] will be used in order to compare these models.

1) The Magic Formula of Pacejka: This model belongs to the empirical approaches category. The tire forces are defined as follows [5]:

$$
Y(X)=y(x)+S_{v}
$$

With:

$$
\left\{\begin{array}{l}
x=X+S_{h} \\
y=D \sin [C \arctan \{B x-E(B x-\arctan (B x))\}]
\end{array}\right.
$$

Where:

- $Y: F_{x}$ (longitudinal force), $F_{y}$ (lateral force) or $M_{z}$ (aligning torque),

- $X: \kappa$ (longitudinal slip) or $\tan \alpha$ (where $\alpha$ is the sideslip angle),

And: 
- $B$ : stiffness factor,

- $C$ : shape factor,

- $D$ : peak value,

- $E$ : curvature factor,

- $S_{H}$ : horizontal shift,

- $S_{V}$ : vertical shift.

Weighting functions are used to take into account the combined slip:

$$
G=D \cos [C \arctan (B x)]
$$

2) The Brush model: As its name may reveal, the model is assimilated to a set of brush bristles (Figure 3).

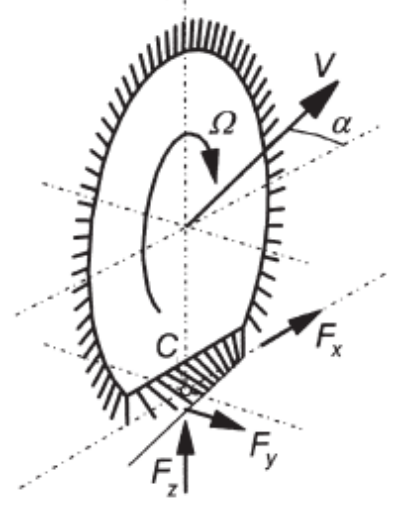

Fig. 3. The Brush model [5].

Several mathematical formulations were adopted regarding this physical representation [4],[5],[7],[8]. These formulas differ according to the assumptions considered regarding the tire physical characteristics, namely, the friction coefficient and the vertical load distribution. In this paper, we suppose that the friction is isotropic and the tire/ground contact surface is rectangular. Longitudinal stiffness is then different from the cornering one. Pursuing the same development logic in [5] and [7], and considering the engine operation instead of braking operation, we find:

- If $\psi\left(\sigma_{x}, \sigma_{y}\right)<1$

$$
\left\{\begin{aligned}
F_{x}\left(\sigma_{x}, \sigma_{y}\right)= & C_{s} \sigma_{x}\left(1-\psi\left(\sigma_{x}, \sigma_{y}\right)\right)^{2} \\
& +\frac{\sigma_{x}}{\sqrt{\sigma_{x}^{2}+\sigma_{y}^{2}}} \mu F_{z} \psi^{2}\left(\sigma_{x}, \sigma_{y}\right) \\
& \left(3-2 \psi\left(\sigma_{x}, \sigma_{y}\right)\right) \\
F_{y}\left(\sigma_{x}, \sigma_{y}\right)= & C_{\alpha} \sigma_{y}\left(1-\psi\left(\sigma_{x}, \sigma_{y}\right)\right)^{2} \\
& +\frac{\sigma_{y}}{\sqrt{\sigma_{x}^{2}+\sigma_{y}^{2}}} \mu F_{z} \psi^{2}\left(\sigma_{x}, \sigma_{y}\right) \\
& \left(3-2 \psi\left(\sigma_{x}, \sigma_{y}\right)\right)
\end{aligned}\right.
$$

- If $\psi\left(\sigma_{x}, \sigma_{y}\right) \geq 1$

$$
\left\{\begin{array}{l}
F_{x}\left(\sigma_{x}, \sigma_{y}\right)=\frac{\sigma_{x}}{\sqrt{\sigma_{x}^{2}+\sigma_{y}^{2}}} \mu F_{z} \\
F_{y}\left(\sigma_{x}, \sigma_{y}\right)=\frac{\sigma_{y}}{\sqrt{\sigma_{x}^{2}+\sigma_{y}^{2}}} \mu F_{z}
\end{array}\right.
$$

Where $\psi\left(\sigma_{x}, \sigma_{y}\right)=\sqrt{\left(\frac{C_{s} \sigma_{x}}{3 \mu F_{z}}\right)^{2}+\left(\frac{C_{\alpha} \sigma_{y}}{3 \mu F_{z}}\right)^{2}}$ represents the adhesion limits when the vertical load distribution is parabolic, and:

- $C_{s} \quad$ : longitudinal stiffness of the tire,

- $C_{\alpha} \quad$ : cornering stiffness of the tire,

- $\mu \quad:$ coefficient of friction,

- $F_{z} \quad \kappa \quad$ : vertical load,

- $\sigma_{x}=\frac{\kappa}{1+\kappa} \quad:$ theoretical longitudinal slip,

- $\sigma_{y}=\frac{\tan (\alpha)}{1+\kappa}:$ theoretical sideslip angle.

3) The physical model of Dugoff: Dugoff developed an analytic model based on the classical analysis of Fiala [6]. He assumed a constant friction coefficient and a constant vertical load distribution. These assumptions give:

$$
\left\{\begin{array}{l}
F_{x}=C_{s} \frac{\kappa}{1-\kappa} \tau \\
F_{y}=C_{\alpha} \frac{\tan (\alpha)}{1-\kappa} \tau
\end{array}\right.
$$

$\tau$ is introduced to take into account the combined slip:

$$
\tau= \begin{cases}(2-\sigma) \sigma & \text { if } \sigma<1 \\ 1 & \text { otherwise }\end{cases}
$$

Where:

$$
\sigma=\frac{(1-\kappa) \mu F_{z}}{2 \sqrt{C_{s}^{2} \kappa^{2}+C_{\alpha}^{2} \tan ^{2}(\alpha)}}
$$

4) Comparison: The Magic Formula is widely used by car manufacturers to simulate and validate dynamic performances due to its precision. Its parameters are changed offline to take into account the different road conditions. Our goal is to develop a model for control synthesis. The different parameters should be changed online. To do this, we should use physical parameters that could be measured or at least estimated. Consequently, we also use the Magic Formula only for validation.

To compare the physical models with Pacejka's, the Magic Formula parameters should be interpreted to meet a physical meaning. In [5], Pacejka pointed out that the product $B C D$ is equivalent to the stiffness. Indeed, considering the equation (3), the product $B C D$ represents the tangent at the origin in the same way as $C_{s}$ for the longitudinal force in equation (5) or (9), and $C_{\alpha}$ for the lateral force in equation (6) or (10). We use the shape coefficients given in [10] for a vertical load of $4000 \mathrm{~N}$, the stiffness coefficients are then given the value of the product $B C D$. Figure 4 depicts the differences for the longitudinal force in a combined slip with a sideslip angle equal to $3^{\circ}$. 


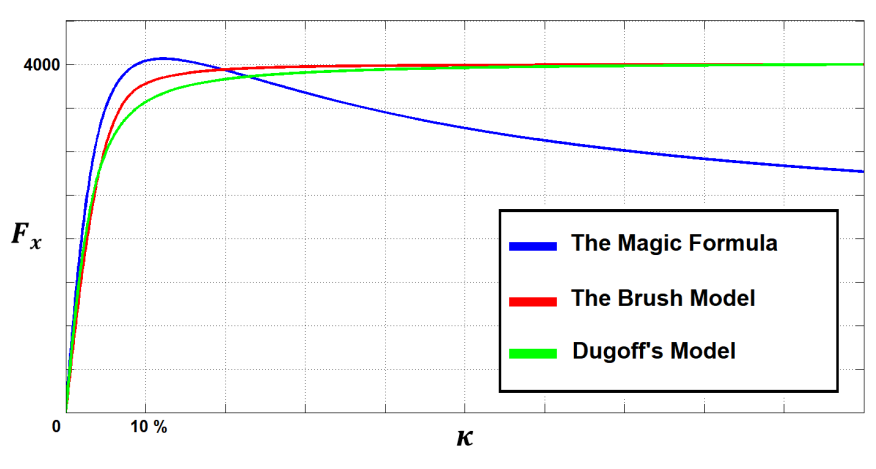

Fig. 4. Comparison of models' longitudinal force shapes in combined slip.

Although the brush model uses physical parameters, it does not take into account the loss of potential phenomenon when maximum friction force is reached. In [7], this model has been improved by considering a speed dependent friction, and in [8], an asymmetric vertical load is considered to improve the peak value location accuracy. However, the complexity also increases.

Dugoff's model suffers from the same drawback. The force's shape is totally inaccurate in the nonlinear region given that the coefficient of friction and the vertical load distribution are considered constant. In [11], the authors developed weighting functions to improve the model in the nonlinear region. Accuracy increases, but the model loses its analytic nature.

In control synthesis, these criteria are not crucial. As we mentioned, the third region of Figure 2 is unstable. There is no point to control the vehicle in this region. This behavior should be predicted and avoided. Thereby, the Brush model and Dugoff's model represent good candidates.

These two models respect three out of the four requirements that we proposed at the beginning of this section. They both respect the tire physical fundamentals, they represent precisely enough the combined slip in the stable region and they are dependent of the physical parameters. However, their mathematical formulas are still too complex to manipulate. They should be linearized.

\section{Tire physical models linearization}

Dugoff's model differs from the brush model by the vertical load distribution assumptions. Dugoff supposes a constant vertical load distribution [6]. In the brush model, a parabolic distribution is considered [7]. Figure 4 shows however that little improvement is brought by this assumption. On the other hand, the formulas (5) and (6) are more complex to manipulate than (9) and (10). The brush model equations' derivatives are too heavy to be exposed. Hence, only Dugoff's model linearization is presented, but both results are illustrated in section III.

According to (11), Dugoff's model has two expressions depending on whether the tire effort has reached its saturation or not. As our concern is the stable part of the tire behavior, it is the expression related to the case where $\sigma<1$ that should be linearized.

1) Longitudinal force linearization: Let $\kappa^{*}$ be the stable operating point located in the linear region of the tire force curve (Figure 2). After replacing the variable $\tau$ in equation (9) by its expression in equation (11), and $\sigma$ by its expression in (12), we perform a first-order Taylor series approximation of $F_{x}$ about $\kappa^{*}$ :

$$
\begin{aligned}
F_{x}(\kappa)= & {\left[F_{x}\left(\kappa^{*}\right)-\left.\frac{d F_{x}\left(\kappa^{*}\right)}{d \kappa}\right|_{\kappa=\kappa^{*}} \kappa^{*}\right] } \\
& +\left.\frac{d F_{x}\left(\kappa^{*}\right)}{d \kappa}\right|_{\kappa=\kappa^{*}} \kappa
\end{aligned}
$$

As the linear region is concerned, $\kappa^{*}$ is very small. We verify analytically that:

$$
\left(\kappa^{*} \ll\right) \Rightarrow\left(\left.F_{x}\left(\kappa^{*}\right) \cong \frac{d F_{x}\left(\kappa^{*}\right)}{d \kappa}\right|_{\kappa=\kappa^{*}} \kappa^{*}\right)
$$

Therefore:

$$
\left(\kappa^{*} \ll\right) \Rightarrow\left(F_{x}(\kappa) \cong \frac{F_{x}\left(\kappa^{*}\right)}{\kappa^{*}} \kappa\right)
$$

Equation (15) shows that the term $\frac{F_{x}\left(\kappa^{*}\right)}{\kappa^{*}}$ corresponds to a longitudinal stiffness. Let us symbolize it $C_{s}^{*}$. Using Dugoff's model equations (9), (11), and (12), we find:

$$
\begin{aligned}
& C_{s}^{*}\left(\alpha, \mu, F_{z}\right) \\
& =\frac{4 \sqrt{C_{s}^{2} \kappa^{*^{2}}+C_{\alpha}^{2} \tan ^{2}(\alpha)}-\left(1-\kappa^{*}\right) \mu F_{z}}{4\left(C_{s}^{2} \kappa^{*^{2}}+C_{\alpha}^{2} \tan ^{2}(\alpha)\right)} \mu F_{z} C_{s}
\end{aligned}
$$

This longitudinal stiffness depends on varying-parameters $\alpha$ (sideslip), $\mu$ (coefficient of friction), and $F_{z}$ (Vertical load). These parameters could be estimated online [4],[12],[13]. It depends also on constant parameters $C_{s}$ (longitudinal stiffness in a non-combined slip), $C_{\alpha}$ (lateral stiffness in a noncombined slip), and $\kappa^{*}$. In section IV, an explicit expression will be given to $\kappa^{*}$. By setting:

$$
\rho=\frac{4 \sqrt{C_{s}^{2} \kappa^{*^{2}}+C_{\alpha}^{2} \tan ^{2}(\alpha)}-\left(1-\kappa^{*}\right) \mu F_{z}}{4\left(C_{s}^{2} \kappa^{*^{2}}+C_{\alpha}^{2} \tan ^{2}(\alpha)\right)} \mu F_{z}
$$

it appears that $C_{s}^{*}$ is the product of $C_{s}$ and a coupling term $\rho$. As a result, the linearized longitudinal force:

$$
F_{x}(\kappa)=C_{s}^{*} \kappa
$$

respect the proposed requirements:

- Respect of the tire physical nature as it depends on $\alpha, \mu$, and $F_{z}$,

- Taking into account the combined slip by means of $\rho$,

- Linearity of the formula and therefore simplicity regarding control issues,

- Dependency on online estimable parameters [4],[12],[13]. 
2) Lateral force linearization: The same reasoning is pursued. Here we suppose an $\alpha^{*}$ to be a stable operating point in the tire linear region. Again, we have $\alpha^{*} \ll$. We proceed then to the same simplifications as (14) and (15). Moreover, in the vicinity of $\alpha^{*}$ :

$$
(\alpha \ll) \Rightarrow(\tan (\alpha) \cong \alpha)
$$

Again, a lateral stiffness $C_{\alpha}^{*}$ can be defined using Dugoff's model equations (10), (11), and (12):

$$
\begin{aligned}
& C_{\alpha}^{*}\left(\kappa, \mu, F_{z}\right) \\
& =\frac{4 \sqrt{C_{s}^{2} \kappa^{2}+C_{\alpha}^{2} \alpha^{*^{2}}}-(1-\kappa) \mu F_{z}}{4\left(C_{s}^{2} \kappa^{2}+C_{\alpha}^{2} \alpha^{*^{2}}\right)} \mu F_{z} C_{\alpha}
\end{aligned}
$$

The linearized lateral force is expressed as:

$$
F_{y}(\alpha)=C_{\alpha}^{*} \alpha
$$

The proposed requirements are again fulfilled. The only difference is that $C_{\alpha}^{*}$ depends on $\kappa$ instead of $\alpha$.

3) Dynamic saturation: The linearized forces developed are indefinitely increasing. As we have mentionned, the overall adhesion is delimited by a "friction ellipse" [5], [7], [10]. This concept is illustrated in Figure 5.

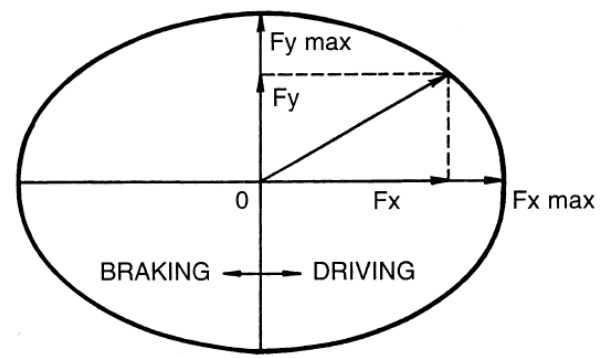

Fig. 5. The friction ellipse concept [10].

The tire potential is delimited by the product $\mu F_{z}$. In general, the friction can be anisotropic. The maximum longitudinal force could be therefore superior to the lateral force maximum. To simplify the problem, we have considered an isotropic friction. Thus, we are facing a "friction circle concept". Despite this simplification, the same logic is applied. When the tire is only solicited longitudinally, the maximum longitudinal force is equal to $\mu F_{z}$. If the tire is solicited laterally while accelerating or braking, the overall force moves along the friction circle. The maximum overall tire force is still $\mu F_{z}$, but the the maximum longitudinal force is penalized. The different forces saturations should be dynamic:

$$
\left\{\begin{array}{l}
F_{x} \leq \sqrt{\left(\mu F_{z}\right)^{2}-F_{y}^{2}} \\
F_{y} \leq \sqrt{\left(\mu F_{z}\right)^{2}-F_{x}^{2}}
\end{array}\right.
$$

\section{Simulation OF THE Linearized MOdel}

By reassembling the equations (16), (18), (20)-(23), we simulate the new linearized tire model and compare it with Pacejka model using the equations (1)-(4) in Matlab/Simulink $^{\complement}$ environment. The linearized Brush-based model is also plotted.

\section{A. Brush-based and Dugoff-based linearized models compar- ison}

Here, we only present the longitudinal force in combined slip with a sideslip angle of $1^{\circ}$ (see Figure 6). The lateral force follows the same behavior with different parameters.

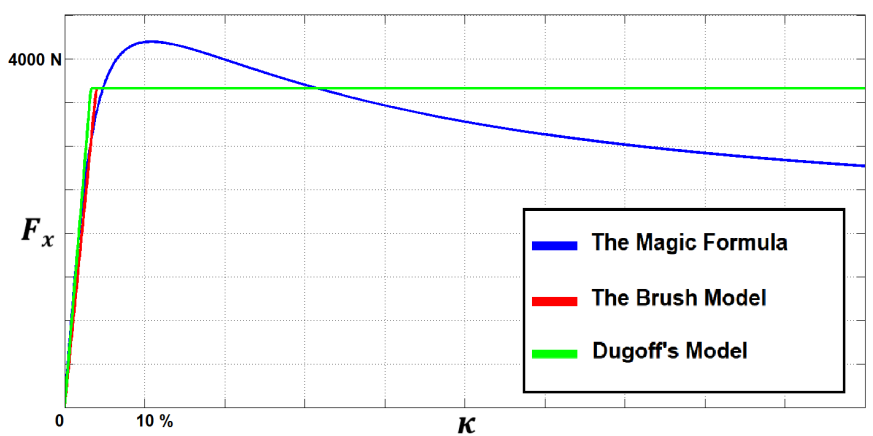

Fig. 6. Comparison of Pacejka model with both linearized models in combined slip.

Although the brush model considers a parabolic vertical load distribution, once linearized it does not offer much more precision with respect to Dugoff's model. The linearized model based on Dugoff's model provides better simplicity/precision compromise. This latter will be then retained.

\section{B. Linearized Dugoff-based model validation}

Note in Figure 6 that the longitudinal force does not reach the maximum of $\mu F_{z}=4000 \mathrm{~N}$ because a part of the tire potential is reserved for the lateral force. The curve's slope of the longitudinal force also varies with the sideslip angle because the longitudinal stiffness varies (see Figure 7).

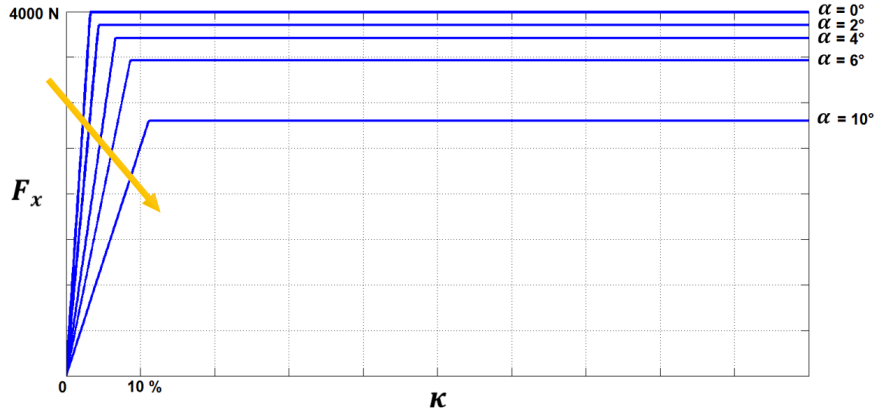

Fig. 7. Variation of the longitudinal force slope with respect to sideslip angle (Linearized Dugoff-based model).

Same remark holds for the lateral force with respect to the longitudinal slip (Fig. 8).

That is to say that the linearized Dugoff-based model represent well the combined slip behavior in the stable region of the tire. More realistic simulations are provided in the next section. 


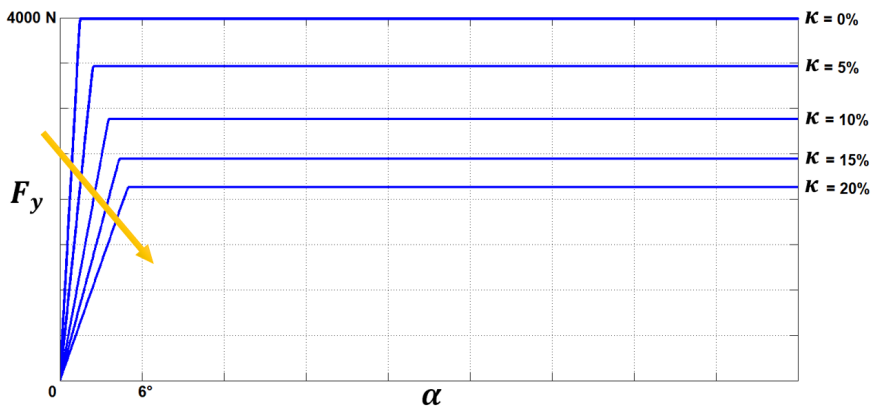

Fig. 8. Variation of the lateral force slope with respect to longitudinal slip (Linearized Dugoff-based model).

\section{RELEVANCE OF THE NEW TIRE MODEL}

In control synthesis, a linear model of the tire has been used in several works [1],[4],[14],[15]. This model has a simpler representation:

$$
\left\{\begin{array}{l}
F_{x}=C_{s} \kappa \\
F_{y}=C_{\alpha} \alpha
\end{array}\right.
$$

The tire behavior is well represented in the linear region, but the model does not take into account the combined slip. This model is more suitable for vehicle stand-alone subsystems. For example, in [14], this simple model is used to develop vehicle longitudinal control logic through a 4 Wheel-Drive Hybrid Electric system. In [16] and [17], a bicycle model, which is based on the simple linear tire model, is used for lateral control performance improvement. With the arrival of autonomous vehicles, the virtual pilot must handle multiobjective control problems. Couplings should be therefore considered, especially at the tire level. For Global Chassis Control (GCC), the linearized Dugoff-based model proposed in this paper is necessary. To illustrate this, a vehicle global dynamic analysis is described next using AMESim ${ }^{\circledR}$ platform.

\section{A. Comparison of linear models}

To compare between the two linear models, another requirement should be added. The new linear tire model should be equivalent to the simpler one when there is no combined slip. This requirement enables giving explicit expressions to the different stable operating points $\kappa^{*}$ and $\alpha^{*}$.

1) $\kappa^{*}$ Calculation: Respecting the previous requirement means:

$$
C_{s}^{*}\left(0, \mu, F_{z}\right)=C_{s}
$$

According to the expression (16), we find:

$$
\kappa^{*}=\frac{\mu F_{z}}{8 C_{s}^{2}}\left[\mu F_{z}+4 C_{s} \pm \sqrt{\left(\mu F_{z}\right)^{2}+8 \mu F_{z} C_{s}}\right]
$$

Both results are acceptable because $\mu F_{z}+4 C_{s} \gg$ $\sqrt{\left(\mu F_{z}\right)^{2}+8 \mu F_{z} C_{s}}$. Here, we consider the highest one.
2) $\alpha^{*}$ Calculation: Again, the previous requirement leads to:

$$
C_{\alpha}^{*}\left(0, \mu, F_{z}\right)=C_{\alpha}
$$

According to the expression (20), we find:

$$
\alpha^{*}=\frac{\mu F_{z}}{2 C_{\alpha}}
$$

3) AMESim $^{\circledR}$ simulation: The AMESim ${ }^{\circledR}$ demo used describes complete chassis model and provide a global dynamics analysis. Inputs are steering angle and engine torque. A combined slip could then be observed. The vehicle is a Bsegment class car. The model is composed of a chassis model with 15 degrees of freedom with assosicated subsystems: elastokinematics module, suspension (spring, damper, lower and higher end stop, antiroll bar), aerodynamic module, tire, road, sensors, powertrain unit, braking system and powersteering system. It is a highly nonlinear global model whose purpose is to represent as precise as possible the vehicle behavior. Most importantly, it uses Pacejka's model to calculate tires' efforts. We add supplementary modules that include the new linear Dugoff-based tire model and the classic linear tire model and compare it with The Magic Formula of Pacejka. Figure 9 illustrates the longitudinal force and Figure 10 illustrates the lateral force.

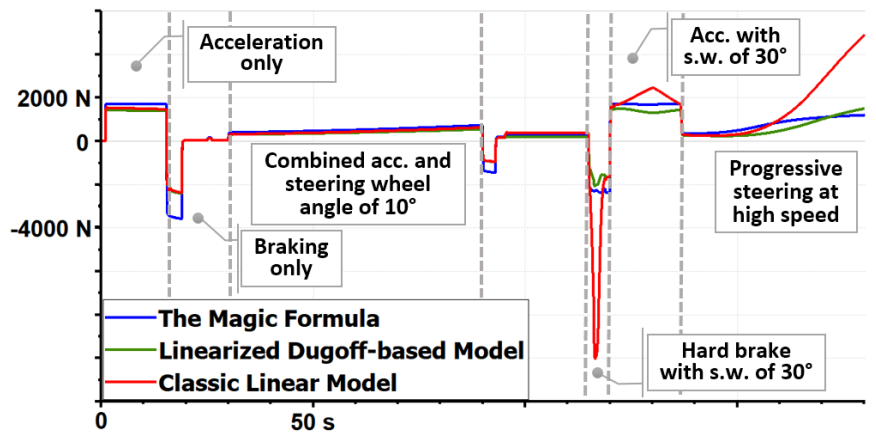

Fig. 9. AMESim ${ }^{\circledR}$ comparison of Pacejka model with linearized models Front left tire longitudinal force.

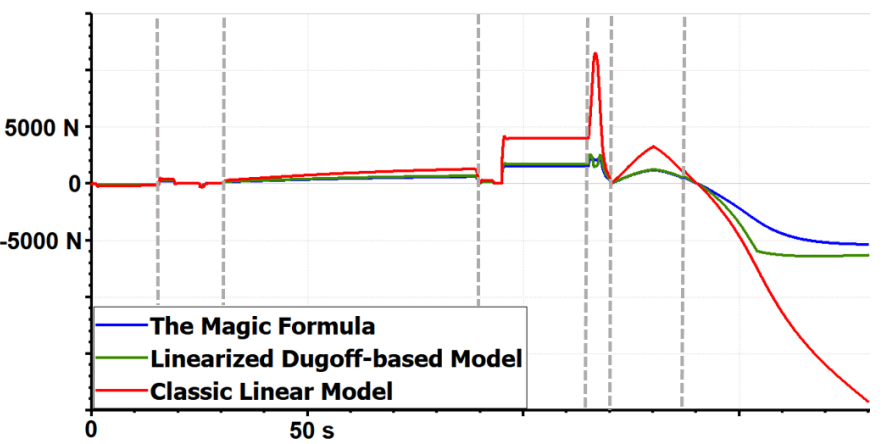

Fig. 10. AMESim ${ }^{\complement}$ comparison of Pacejka model with linearized models Front left tire lateral force.

When only longitudinal force is requested (acceleration or braking), both linear models are close enough to Pacejka's 
model. The lateral force of the classic linear model starts to deviate as soon as the tire is solicited longitudinally and laterally at the same time. Both longitudinal and lateral forces of the classic linear model diverge at severe conditions, for example in a hard brake maneuver while steering or progressive steering at high velocity. In contrast, the new linearized model follows close enough the Magic Formula. When the tire is solicited longitudinally and laterally at the same time, both longitudinal and lateral stiffnesses are updated and dynamic saturations are recalculated. The values of the forces are then lower.

In control problems, this is crucial. In fact, ignoring combined slip may lead to high tire force demand. This could exceed the saturated value and destabilize the vehicle. The new linearized model proposed in this paper aims preventing this situation.

\section{B. Further improvements}

So far, all mathematical formulas developed are expressed in the steady state. In control synthesis, time response is an important criterion. Hence, the transient behavior should be included.

1) Relaxation length: Tire force delay is caused by carcass deflections [5],[7]. This is usually represented by a first order lag [18],[19]:

$$
F_{i}=\frac{1}{1+\tau_{i} s} F_{i}^{s s}
$$

$i$ is either $x$ or $y, \tau_{i}$ is the time-constant, $s$ is the Laplace operator, and $F_{i}^{s s}$ is the steady-state force that have been developed in the previous sections.

The carcass deflections change with the vehicle speed [5],[7],[18],[19]. Time-constant in equation (30) depends then on vehicle speed:

$$
\tau_{i}=\frac{3 L_{i}}{V_{x}}
$$

$L_{i}$ is the relaxation length and $V_{x}$ is the vehicle longitudinal speed. The relaxation length represent the distance that tire rolls before the lateral force reaches $63 \%$ of its final value [5]. This does not influence much the shape of the tire response as the Fig. 11 shows.

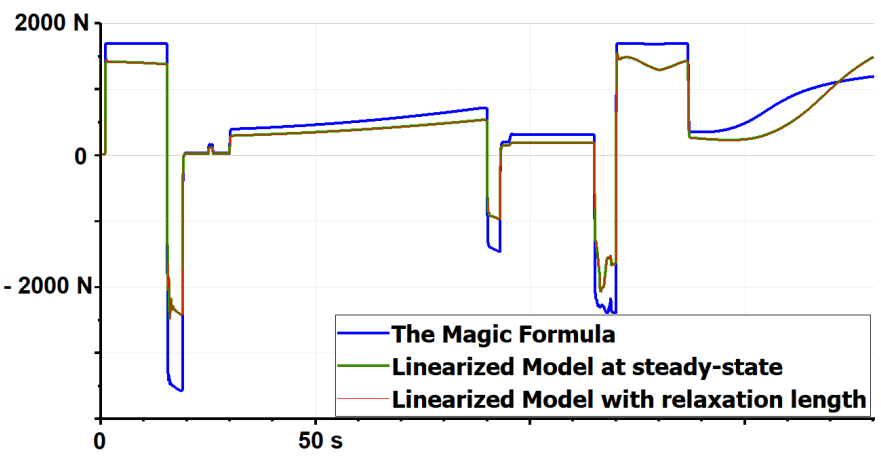

Fig. 11. AMESim $\left.{ }^{(}\right)$comparison of Pacejka model and linearized models with and without transient behavior - Front left tire longitudinal force.
This is mainly due to the low value of the relaxation length (around $0.02 \mathrm{~m}$ ). In control synthesis, the transient behavior could be ignored to prevent high order controllers design.

\section{Controllability}

The linearized model is developed for control synthesis suitability. A state-space representation can be proposed:

$$
\left\{\begin{array}{l}
\dot{x}(t)=A(t) x(t)+B(t) u(t) \\
y(t)=C(t) x(t)
\end{array}\right.
$$

Where:

- $x(t)=\left[\begin{array}{c}F_{x} \\ F_{y}\end{array}\right] \quad:$ is the state vector,

- $y(t)=\left[\begin{array}{c}F_{x} \\ F_{y}\end{array}\right] \quad:$ is the output vector,

- $u(t)=\left[\begin{array}{l}\kappa \\ \alpha\end{array}\right] \quad:$ is the input vector,

- $A(t)=\left[\begin{array}{cc}-\frac{1}{\tau_{x}} & 0 \\ 0 & -\frac{1}{\tau_{y}}\end{array}\right]$ : is the state matrix,

- $B(t)=\left[\begin{array}{cc}\frac{C_{s}^{*}}{\tau_{x}} & 0 \\ 0 & \frac{C_{\alpha}^{*}}{\tau_{y}}\end{array}\right] \quad:$ is the input matrix,

- $C(t)=\left[\begin{array}{ll}1 & 0 \\ 0 & 1\end{array}\right] \quad:$ is the output matrix.

Without forgetting the constraints (22) and (23).

Note that both state and input matrices contain varying parameters. Although stiffness coefficients depend on input variables, they cannot be splitted as we need the whole expression to be multiplied by its corresponding input variable.

According to the structure of the matrices $A, B$ and $C$, the system is clearly stable, controllable and observable as long as the varying parameters are positive, which is the case.

Finally, this representation is suitable for modular and flexible control architectures. A simple tire module can be added as the Fig. 12 shows where the tire block contains equations (32) and (33) and constraints (22) and (23).

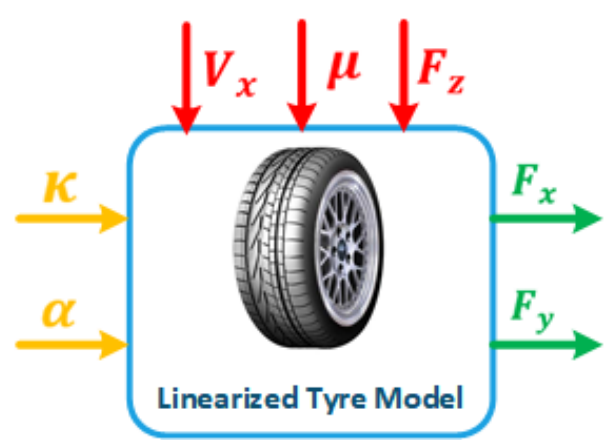

Fig. 12. Linearized tire module with varying-parameters. 


\section{Discussion and application}

We recall that this model was developed to face Global Chassis Control issues. As far as the overall chassis is concerned, coordination is a key aspect. Several techniques exist in this context. For example, rule-based strategies using fuzzy logic control downstream the standalone subsystems have been used in [20]. Autonomous vehicles require additional subsystems. As the number of subsystems increases, development of such strategies becomes less obvious. Optimization techniques performed upstream the standalone subsystems present a promising alternative in this situation. Moreover, today's vehicles are over-actuated [1]. That is to say that subsystems control outputs exceed the number of control axes of the vehicle. The solution is therefore not unique. This should not be seen as a disadvantage, but rather as an opportunity to introduce secondary objectives. In this framework, control allocation techniques present more suitable solutions [21].

Control allocation problems are defined as follow [22]: find the control vector, $\vec{\delta} \in \mathbb{R}^{n}$ such that

$$
\mathbf{B} \vec{\delta}=\vec{d}_{d e s}
$$

subject to

$$
\left\{\begin{aligned}
& \vec{\delta}_{\min } \leq \vec{\delta} \leq \vec{\delta}_{\max } \\
& \dot{\vec{\delta}} \leq \dot{\vec{\delta}}_{\max }
\end{aligned}\right.
$$

where $\mathbf{B} \in \mathbb{R}^{n \times m}$ is a control effectiveness matrix, $\vec{\delta}_{\text {min }}$ and $\vec{\delta}_{\text {max }}$ are the lower and upper position limits respectively, $\dot{\vec{\delta}}$ is the control rate, $\dot{\vec{\delta}}_{\text {max }}$ is the maximum control rate, $\vec{d}_{d e s}$ are the desired accelerations, $n$ is the number of control effectors, and $m$ is the number of axes to control $(n>m)$.

For ground vehicles, tires are the sole effectors. Distribution of control commands is mainly constrained by tires' potential. As far as combined slip is concerned, this potential varies. Tires' stiffness and maximum efforts should be updated. For example, in equation $(22), F_{x}$ is constrained by the overall potential $\left(\mu F_{z}\right)$ and also $F_{y}$. The lateral stiffness should be updated by taking into account the friction ellipse concept. In this way, we can favour the tires with greater potential.

\section{CONCLUSION}

A new linear tire model with varying parameters has been developed in this paper. This model is particularly suitable for control problems and especially for global chassis control. Unlike other linear models, our model takes into account the combined slip in the tire's stable region where control is justified. It depends also on physical parameters that can be estimated, which is necessary for online operations.

More evidence is required to validate this model. Unfortunately, experiments were not carried out because they are too tedious for a simple model validation. Our future works will rather focus on the model ability to generate safer control strategies regarding coordinated control for global chassis control.

\section{ACKNOWLEDGMENT}

The authors would like to thank Dr. Xavier Mouton from the Group Renault for his supervision of the Vehicle Motion Control (VMC) project.

\section{REFERENCES}

[1] M. A. Selby, "Intelligent Vehicle Motion Control," PhD thesis, University of Leeds, Feb. 2003.

[2] S. Brennan and A. Alleyne, "Integrated vehicle control via coordinated steering and wheel torque inputs," in American Control Conference, (2001), pp.7-12.

[3] M. Velardocchia and A. Vigliani, "Control systems integration for enhanced vehicle dynamics," in The Open Mech. Eng. J., vol. 7, 2013, pp. 58-69.

[4] A. Soltani, "Low Cost Integration of Electric Power-Assisted Steering (EPAS) with Enhanced Stability Program (ESP)," PhD thesis, Cranfield University, 2014.

[5] H.B. Pacejka, "Tyre and Vehicle Dynamics," Second Edition. Elsevier, Butterworth-Heinemann, 2006.

[6] H. Dugoff, P. Fancher and L. Segel, "Tire Performance Characteristics Affecting Vehicle Response to Steering and Braking Control Inputs," Highway Safety Research Institute of Science and Technology, The University of Michigan, Michigan, technical report, CST 460, Aug 1969.

[7] J. Svendenius, "Tire Models for Use in Braking Applications," Department of Automatic Control, Lund Institute of Technology, 2003.

[8] J. Svendenius and B. Wittenmark, "Brush Tire Model with Increased Flexibility," in 2003 European Control Conference (ECC), Cambridge, UK, 2003, pp. 1863-1868.

[9] Michelin, "Le pneu: L'adhrence," Clermont-Ferrand, France, Janvier 2001.

[10] J. Y. Wong, "Theory of Ground Vehicles, 3rd Edition, John Wiley \& Sons, 2001

[11] M. Bian, L. Chen, Y. Luo, and K. Li, "A Dynamic Model for Tire/Road Friction Estimation under Combined Longitudinal/Lateral Slip Situation," in SAE Technical Paper 2014-01-0123, 2014.

[12] J. Svendenius, "Tire Modeling and Friction Estimation, Department of Automatic Control, Lund University, 2007.

[13] K. B. Singh and S. Taheri, "Estimation of tireroad friction coefficient and its application in chassis control systems," in Systems Science \& Control Engineering, pp. 39-61, 2015.

[14] Lu Xiong and Zhuoping Yu, "Research on Robust Control for longitudinal Impact of 4 Wheel-Drive Hybrid Electric Vehicle," in Procedia Engineering, vol. 15, pp. 293-297, 2011.

[15] T. Bchle, K. Graichen, M. Buchholz, and K. Dietmayer, "SlipConstrained Model Predictive Control Allocation for an All-Wheel Driven Electric Vehicle," in IFAC Proceedings Volumes, vol. 47, issue 3, pp. 12042-12047, 2014.

[16] M. Harada and H. Harada, "Analysis of lateral stability with integrated control of suspension and steering systems", in JSAE Review, vol. 20, issue 4, pp. 465-470, 1999.

[17] Z. Shuai, H. Zhang, J. Wang, J. Li, and M. Ouyang, "Lateral motion control for four-wheel-independent-drive electric vehicles using optimal torque allocation and dynamic message priority scheduling", in Control Engineering Practice, vol. 24, pp. 55-66, 2014.

[18] Raffaele Di Martino, "Modelling and simulation of the dynamic behaviour of the automobilel," PhD thesis, Universit de Haute Alsace Mulhouse, 2005.

[19] M. Klomp and M. Lidberg, "Safety Margin Estimation in Steady State Maneuvers", in Proceedings of the 8th International Symposium on Advanced Vehicle Control, 2006.

[20] J.-X. Wang, N. Chen, D.-W. Pi and G.-D. Yin, "Agent-based coordination framework for integrated vehicle chassis control," in Proc. Inst. Mech. Eng., D, J. Automobile Eng., vol. 223, no. 5, pp. 601-621, 2009.

[21] T. A. Johansen and T. I. Fossen, "Control Allocation - A survey," in Automatica, Vol. 49, Issue 5, May 2013, pp. 10871103.

[22] M. W. Oppenheimer, D. B. Doman, and M. A. Bolender, "Control Allocation for Over-actuated Systems," in 2006 14th Mediterranean Conference on Control and Automation, Ancona, 2006, pp. 1-6. 